\section{A simplified method for differential staining of aborted and non-aborted pollen grains}

\author{
Ross Peterson,' Janet P Slovin, ${ }^{2}$ \\ Changbin Chen'
}

'Department of Horticultural Science, University of Minnesota, MN, USA;

${ }^{2}$ Genetic Improvement of Fruits and Vegetables Laboratory, United States Department of Agriculture, Baltimore, MD, USA

\begin{abstract}
The ability to use chemical staining to discriminate aborted from non-aborted pollen grains has well-known practical applications in agriculture. A commonly used technique for assessing pollen vitality, Alexander's stain, uses chloral hydrate, phenol and mercuric chloride, all of which are highly toxic. We describe here an improved pollen staining technique that avoids the use of a regulated chemical chloral hydrate and two extremely toxic chemicals mercuric chloride and phenol, and requires a much shorter time period for sample preparation and staining. This simplified method is very useful for field studies without high-end equipments such as fluorescence microscopes. Samples can be collected and fixed in the fields and examined in a simple laboratory that has light microscopes.
\end{abstract}

\section{Introduction}

A facile assay for determination of the vitality of pollen grains is important for plant breeders as well as for biologists interested in development of the male gametophyte. Pollen germination assays frequently require optimization as well as being time consuming and difficult to reproduce. ${ }^{1}$ A simple rapid staining technique that clearly differentiates aborted from non-aborted pollen would provide needed information and improve throughput. For many years, nuclear dyes such as acetocarmine and cotton blue have been used for staining pollen, as have the vital dyes triphenyl tetrazolium chloride (TTC) and tetrazolium red. TTC and other vital dyes have been found to stain aborted pollen grains to varying degrees. ${ }^{2-4}$ Some fluorescent dyes have been used to differentiate aborted and non-aborted pollen grains, which include a most commonly used dye of fluores- cein diacetate that was initially developed to examine the viability of mammalian cells. ${ }^{5,6}$ The uses of fluorescent dyes, however, require special equipment, such as fluorescence microscopes, which limit the application for field studies and most agricultural extension stations that do not have the available equipment.

A major improvement in differential pollen staining was reported by M.P. Alexander in 1969. Alexander's stain colors aborted pollen grains from most angiosperms and the spores of gymnosperms blue-green, and nonaborted pollen grains and spores stain magenta-red. ${ }^{7}$ Unfortunately, three ingredients of Alexander's protocol exhibit possible health risks. Two of these reagents are chloral hydrate that is used in the stain solution and the mercuric chloride that is used in the fixative. Both are either highly regulated by worldwide government standards on chemical toxicities or threaten the health of those who use the stain solution. ${ }^{8}$ The third harmful reagent is phenol. It can be absorbed through the skin and has shown to disrupt the function of the central nervous system. ${ }^{9}$ Another drawback to Alexander's protocol is the long time it requires to obtain clear results. Thus, it is not a very efficient method and does not allow for acquisition of data in a timely manner. In addition, the protocol calls for optimization of the recipes to penetrate oily, spiny, thick, or thin coatings around the pollen and for staining pollen in non-dehiscent anthers. Due to the protocol variations, experimental results obtained in different laboratories have proven to be inconsistent. Some researchers have obtained excellent results, with differential coloration reflecting the results in Alexander's original report. ${ }^{10-12}$ In some cases, the pollen grains were able to be distinguished, but the images were not clear due to non-transparent anther walls. ${ }^{13}$ In other cases, the anther wall was sufficiently transparent and the non-aborted pollen grains stained red, but the aborted pollen grains were colorless or only very pale green. ${ }^{14-16}$ Such variability can be explained by differences in preparation of the tissues, differences in local environmental conditions during staining, or differences in the source of chemical ingredients.

One additional problem with Alexander's protocol is limited access to chloral hydrate, which requires restricted federal and state licenses for purchase in the United States, that also incur additional costs to the research laboratory. We report here a simplified staining protocol that does not use chloral hydrate, mercuric chloride or phenol and yet rapidly produces a clear differentiation between aborted and non-aborted pollen, even within non-dehiscent anthers.
Correspondence: Changbin Chen, Department of Horticultural Science, University of Minnesota 380 Alderman Hall, 1970 Folwell Avenue, St. Paul, MN 55108, USA. E-mail: chenx481@umn.edu

Key words: pollen staining, Alexander's stain, chloral hydrate, phenol.

Contributions: RP did most of experiments and contributed to the writing; JPS did further test and contributed to edit the manuscript; CC initiated this study, supervised RP for his bench-work and data collection and wrote this manuscript.

Acknowledgements: we deeply appreciated the comments from all three reviewers, which greatly improved the final protocol with the removal of phenol. The authors are grateful to Dr. Luca Comai for providing Arabidopsis autotetraploid seeds and Dr. Dong-Hoon Jeong for providing rice Nipponbare seeds. Our sincere thanks also go to Tao Li and Asmita Batajoo for plant care, Dr. Junhua Li and the former colleagues, Drs. Cary $\mathrm{H}$ Hord, Yoshitaka Azumi and Wuxing Li at the Pennsylvania State University for sharing their experiences with us. This study was supported by funds from the American Society of Plant Biologists SURF program and the University of Minnesota UROP program to RP and by funds from the USDABRDC, Dow Agrosciences, and University of Minnesota Academic Health Center to CC.

Conflict of interest: the authors report no conflicts of interest.

Received for publication: 19 March 2010.

Revision received: 16 July 2010.

Accepted for publication: 16 July 2010.

This work is licensed under a Creative Commons Attribution 3.0 License (by-nc 3.0).

(C) Copyright R. Peterson et al., 2010 Licensee PAGEPress, Italy

International Journal of Plant Biology 2010; 1:e13 doi:10.4081/pb.2010.e13

\section{Materials and Methods}

\section{Plants}

A total of 22 plant species and 3 different Arabidopsis lines were tested (Table 1). Arabidopsis, strawberry (Fragaria vesca Coville), tomato (Solanum lycopersicum L.) and rice (Oryza sativa L.) plants were grown in the greenhouse or growth chambers. Other plants tested were grown outdoors under ambient conditions in Minnesota, USA.

\section{Bud fixation}

Flower buds or free anthers were collected before anthesis, when pollen was mature but anthers non-dehiscent (stage 12 flower buds) ${ }^{17}$ 
and fixed in Carnoy's fixative (6 alcohol:3 chloroform: 1 acetic acid $)^{18}$ for a minimum of 2 hours. Buds can be stored in fixative for 12 months at either room temperature or cold room. For large buds, such as Rhododendron, Tulipa or Magnolia, individual anthers were dissected from the stage 13 flower bud and fixed.

\section{Stain solution}

The final stain solution we used was prepared by adding the following constituents in the order given below and stored in the dark.

$10 \mathrm{~mL} 95 \%$ alcohol

$1 \mathrm{~mL}$ Malachite green (1\% solution in 95\% alcohol)

$50 \mathrm{~mL}$ Distilled water

$25 \mathrm{~mL}$ Glycerol

$5 \mathrm{~mL}$ Acid fuchsin (1\% solution in water)

$0.5 \mathrm{~mL}$ Orange $\mathrm{G}$ ( $1 \%$ solution in water)

$4 \mathrm{~mL}$ Glacial acetic acid

Add distilled water $(4.5 \mathrm{~mL})$ to a total of $100 \mathrm{~mL}$.

\section{Staining}

Following at least two hours of fixation, the bud can be placed on a microscope slide and the fixative's liquid was thoroughly and carefully dried from the plant material with absorbent paper. Proper safety gloves should be worn to avoid the risk of chloroform from being absorbed through the skin. Apply 2-4 drops of the stain solution before the sample completely dries. If flower buds have been collected instead of free anthers, the buds should be dissected to release the anthers and pollen. Under a dissecting microscope the leftover plant debris can be carefully removed. To save stain solution, samples can be dissected prior to putting individual anthers into stain. Some anthers, such as those of Magnolias, are too large to be viewed intact and must be dissected further.

Once the sample is in the stain, slowly heat the slide over an alcohol burner in a fume hood until the stain solution is near boiling ( $\sim 30$ seconds). A more moderate rate of heating allows better penetration of the dye into the cellulose and protoplasm of the pollen. Extremely high temperatures resulting in smoking or bubbling of the stain can burn the dye and the sample. Heating can be adjusted by briefly moving the slide in and out of the flame. To ensure stain has been completely absorbed into the pollen grains, 10 to 15 minutes should be allowed for some species such as Lonicera tatarica, Ginkgo biloba, Pinus resinosa and Rhododendron mucronulatum.

\section{Imaging}

Place a cover-slip over the sample and apply even pressure on the cover-slip to ensure that all plant components converge to one plane. The cover-slip can be sealed using nail polish or wax. Slides were examined using a Leitz microscope (Ernst Leitz Wetzlar GmbH, Germany). Micrographs were taken using a Spot Insight digital camera (Diagnostic instruments, Inc. Sterling Heights, MI, USA) and edited with Adobe Photoshop CS2 (Adobe Systems Inc. CA, USA).

\section{Results and Discussion}

The simplified stain resulted in excellent differentiation of pollen in all 22-plant species tested (Table 1). Photographs of 13 out of 22 examined species are included in Figure 1, all of which demonstrated clear differentiation of aborted and non-aborted pollen grains, except the Arabidopsis Ler ecotype that showed all non-aborted pollen grains (Figure 1D). To represent different types of pollen and/or anther structures, both Angiosperms and Gymno sperms were studied. To exemplify these different morphologies certain species are shown in Figure 1. Of the Angiosperm species, strawberry [Fragaria vesca (Rugen)] has thick

Table 1. A list of plant species tested.

\begin{tabular}{|c|c|c|c|c|}
\hline Divisions & $\begin{array}{l}\text { Species name } \\
\text { (family) }\end{array}$ & Fig. & $\begin{array}{l}\text { Non-aborted } \\
\text { pollen }(\%)^{*}\end{array}$ & $\begin{array}{l}\text { Non-aborted } \\
\text { pollen }(\%)^{* *}\end{array}$ \\
\hline \multirow[t]{2}{*}{ Gymnosperm } & Ginkgo biloba L. (Ginkgoaceae) & $1 \mathrm{~A}$ & 94.5 & 97.5 \\
\hline & Pinus resinosa Ait. (Pinaceae) & 1B & 95.5 & 93.0 \\
\hline \multirow[t]{20}{*}{ Angiosperm } & Acer negundo L. (Aceraceae) & N/A & 95.5 & 96.0 \\
\hline & Acer rubrum L. (Aceraceae) & $1 \mathrm{C}, 1 \mathrm{~F}$ & 99.0 & 98.5 \\
\hline & Alnus incana (L.) Moench. (Betulaceae) & $\mathrm{N} / \mathrm{A}$ & 92.5 & 90.5 \\
\hline & $\begin{array}{l}\text { Arabidopsis thaliana (L.) } \\
\text { Heynh. (Ler ecotype: diploid, } \\
\text { tetraploid, and } d m c 1 \text { mutants) } \\
\text { (Brassicaceae) }\end{array}$ & $\begin{array}{l}1 \mathrm{D} \\
1 \mathrm{E} \\
\mathrm{N} / \mathrm{A}\end{array}$ & $\begin{array}{l}98.5 \\
66.5 \\
15.5\end{array}$ & $\begin{array}{l}95.5 \\
72.5 \\
22.0\end{array}$ \\
\hline & Berberis thunbergi DC. (Berberidaceae) & $\mathrm{N} / \mathrm{A}$ & 87.0 & 98.5 \\
\hline & Betula populifolia Marsh. (Betulaceae) & $1 \mathrm{G}$ & 97.5 & 99.0 \\
\hline & Fragaria vesca Coville cv. Rugen (Rosaceae) & $1 \mathrm{H}$ & 83.5 & 82.0 \\
\hline & Lonicera tatarica L. (Caprifoliaceae) & $1 I$ & 90.5 & 93.5 \\
\hline & $\begin{array}{l}\text { Magnolia stellata (Siebold \& Zucc.) } \\
\text { Maxim. (Magnoliaceae) }\end{array}$ & $\mathrm{N} / \mathrm{A}$ & 90.5 & 95.0 \\
\hline & $\begin{array}{l}\text { Malus floribunda Siebold ex Van Houtte } \\
\text { (Rosaceae) }\end{array}$ & $\mathrm{N} / \mathrm{A}$ & 94.0 & 94.5 \\
\hline & Narcissus spp. L. (Amarylliaceae) & N/A & 95.0 & 92.5 \\
\hline & Oryza sativa L. cv. Nipponbare (Poaceae) & $1 \mathrm{~J}$ & 93.0 & 91.0 \\
\hline & $\begin{array}{l}\text { Populus deltoids W. Bartram ex Marshall } \\
\text { (Salicaceae) }\end{array}$ & N/A & 96.5 & 97.0 \\
\hline & Prunus padus L. (Rosaceae) & $1 \mathrm{~K}$ & 90.5 & 88.5 \\
\hline & Quercus rubra L. (Fagaceae) & $\mathrm{N} / \mathrm{A}$ & 81.0 & 80.0 \\
\hline & $\begin{array}{l}\text { Rhododendron mucronulatum Turcz. } \\
\text { (Ericaceae) }\end{array}$ & $1 \mathrm{~L}$ & 64.5 & 82.5 \\
\hline & Scilla siberica Haw. (Liliaceae) & $\mathrm{N} / \mathrm{A}$ & 92.0 & 91.0 \\
\hline & Solanum lycopersicum L. (Solanaceae) & $1 \mathrm{M}$ & 76.5 & 83.5 \\
\hline & Taraxacum officinale F.H. Wigg. (Asteraceae) & $1 \mathrm{~N}$ & 73.5 & 80.0 \\
\hline & Tulipa spp. L. (Liliaceae) & 10 & 52.5 & 64.0 \\
\hline
\end{tabular}

*The stain without phenol; **the stain with phenol. anther walls and small pollen grains (Figure 1H); woody honeysuckle (Lonicera tatarica) has thick pollen walls (Figure 1I); azalea (Rhododendron mucronulatum) shows tetrad pollen structure (Figure 1L); rice [Oryza sativa (Nipponbare)] has thin pollen walls (Figure 1J); dandelion (Taraxacum officinale) has thick and oily pollen walls (Figure 1N) and tulip (Tulipa spp) has larger pollen grains (Figure 10). In all tested samples, non-aborted pollen grains stained magenta-red and aborted pollen grains stained blue-green. This differentiation can be seen in Arabidopsis autotetraploid (Figure 1E). No significant differences in the stain clarity were observed with thick or thin walled pollen grains, or with oily or waxy pollen. Pollen within non-dehiscent anthers (Figure $1 \mathrm{C}, \mathrm{D}, \mathrm{E}, \mathrm{H}, \mathrm{K}, \mathrm{N}$ ) stained as well as free pollen (Figure 1 A, B, G, I), even without the mercuric chloride and phenol used in the original Alexander's staining preparation. ${ }^{7,19}$

As a supplementary improvement for the safety and accessibility of this improved staining technique, phenol was avoided. The origi- 


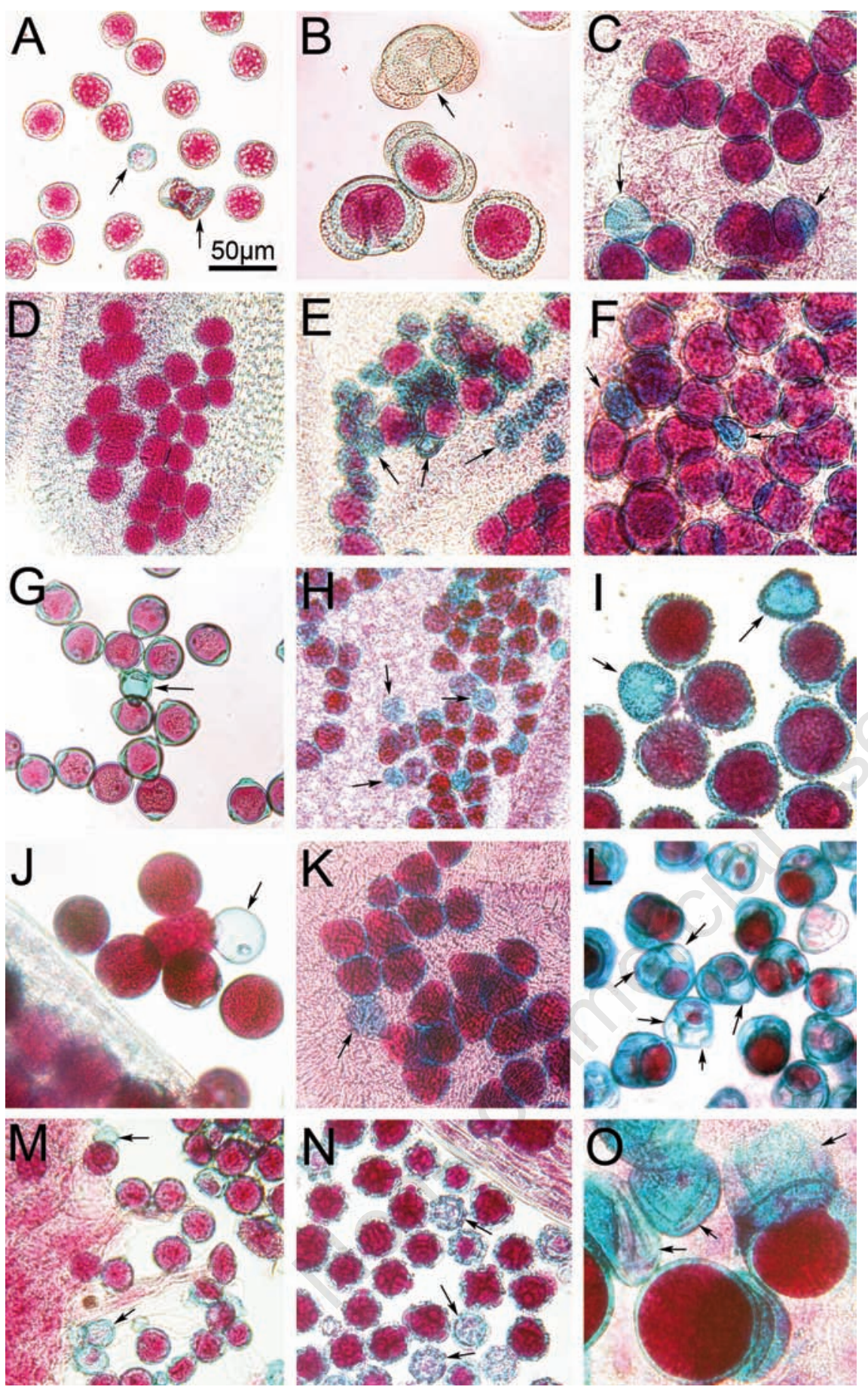

Figure 1. Differential staining of aborted and non-aborted pollen grains. (A) Ginkgo biloba. (B) Pinus resinosa. (C) Acer rubrum, stained without phenol. (D) Arabidopsis thaliana Ler (diploid). (E) Arabidopsis thaliana Ler (autotetraploid). (F) Acer rubrum, stained with phenol. (G) Betula populifolia. (H) Fragaria versca (Rugen). (I) Lonicera tatarica. (J) Oryza sativa. (K) Prunus padus. (L) Rhododendron mucronulatum. (M) Solanum lycopersicum (Microtom). (N) Taraxacum officinale. (O) Tulipa spp. $\mathrm{Bar}=50 \mu \mathrm{m}$, all images are at the same magnification. Arrows indicate aborted pollen grains that stained blue-green. Non-aborted pollen grains are stained magenta-red.

nal use of phenol (5\%) was to help make the stain on the slides more transparent. As a result of this transparency, the differential staining would theoretically become clearer. However, once quantitative and qualitative experiments were done in the absence of phenol, it became evident that phenol was unnecessary for clear differential staining (Figure 1 C, F). A minor difference was noted only for pollens inside the non-dehisced anthers.
Despite this small difference, no significant change of the staining of non-aborted pollen grains was observed when comparing the stains with or without phenol (as shown in Table 1). These data agree with the notion that the stain without phenol is as credible as the stain with phenol. Thus, the omission of phenol in this pollen staining technique is recommended.

Alexander's protocol for differential staining of aborted and non-aborted pollen is a frequently cited technique because of its usability for many different applications. However, the availability of chemicals used for the stain, the variability inherent in protocols that need to be optimized for each plant and the amount of time required to obtain results, limit the protocol's applicability and the reproducibility. In particular, the need to use chloral hydrate requires licenses that may be difficult or expensive to obtain in some countries. The simplified stain described here, using the stain solution with Carnoy's fixative, allows for the use of the protocol in both laboratory and field studies. The procedure can be used for all types of pollen grains. The 2-hour incubation in the chloroform and acetic acid containing fixative sufficiently removes oils or sticky materials and clears pollen and anther walls. Additional heating (1-2 min.) or incubation time (10-15 min.) in the stain, for certain species listed previously, may be necessary for optimal stain absorption. There is no additional chemicals (chloral hydrate or phenol) needed to clear the sample, and no specific fixative needed either. The simplification to using Carnoy's fixative also allows for longer fixation prior to staining, facilitating field studies or studies with large numbers of samples. The entire procedure takes a little more than 2 hours, eliminating the long incubations and the optimization of steps required in the Alexander's stain. ${ }^{7,20}$

\section{References}

1. Boavida LC, McCormick S. Temperature as a determinant factor for increased and reproducible in vitro pollen germination in Arabidopsis thaliana. Plant J 2007;52:570-82.

2. Oberle G, D., Watson R. The use of 2,3,5triphenyl tetrazolium chloride in viability test of fruit pollen. Amer Soc Hort Sci Proc 1953;61:299-303.

3. Aslam LM, Brown MS, Kohel RJ. Evaluation of seven tetrazolium salts as vital pollen stain in Gossypium. Crop Sci 1964;4:508-10.

4. Sarvella P. Vital stain testing of pollen viability. J Hered 1964;55:154-8.

5. Rotman B, Papermaster BW. Membrane properties of living mammalian cells as 
studied by enzymatic hydrolysis of fluorogenic esters. Proc Natl Acad Sci USA 1966;55:134-41.

6. Wang Z-Y, Ge Y, Scott M, Spangenberg G. Viability and longevity of pollen from transgenic and nontransgenic tall fescue (Festuca arundinacea) (Poaceae) plants. Am J Bot 2004;91:523-30.

7. Alexander MP. Differential staining of aborted and nonaborted pollen. Stain Technol 1969;44:117-22.

8. Liedl BE, McCormick S, Mutschler MA. A clearing technique for histochemical location of GUS activity in pullen tubes and ovules of Lycopersicon. Plant Mol Biol Rep 1993;11:194-201.

9. Hathaway GJ, Proctor NH, Hughes JP, Fischman ML. Proctor and Hughes' chemical hazards of the workplace. 3rd ed. New York: Van Nostrand Reinhold 1991.

10. Bleuyard JY, White CI. The Arabidopsis homologue of Xrcc3 plays an essential role in meiosis. EMBO J 2004;23:439-49.

11. Kaur J, Sebastian J, Siddiqi I. The Arabidopsis-mei2-like genes play a role in meiosis and vegetative growth in Arabidopsis. Plant Cell 2006;18:545-59.

12. Chen C, Zhang W, Timofejeva L, et al. The Arabidopsis ROCK-N-ROLLERS gene encodes a homolog of the yeast ATPdependent DNA helicase MER3 and is required for normal meiotic crossover formation. Plant J 2005;43:321-34.

13. Azumi Y, Liu D, Zhao D, et al. Homolog interaction during meiotic prophase I in Arabidopsis requires the SOLO DANCERS gene encoding a novel cyclin-like protein. EMB0 J 2002;21:3081-95.[PubMed]14.

Ebel C, Mariconti L, Gruissem W. Plant retinoblastoma homologues control nuclear proliferation in the female gametophyte. Nature 2004;429:776-80.
15. Grelon M, Vezon D, Gendrot G, Pelletier G. AtSP011-1 is necessary for efficient meiotic recombination in plants. EMBO J. 2001;20:589-600.

16. Hord CL, Sun YJ, Pillitteri LJ, et al. Regulation of Arabidopsis early anther development by the mitogen-activated protein kinases, MPK3 and MPK6, and the ERECTA and related recepter-like kinases. Mol plant 2008;1:645-58.

17. Smyth DR, Bowman JL, Meyerowitz EM. Early flower development in Arabidopsis. Plant Cell 1990;2:755-67.

18. Carnoy JB. Les globules polaires de 1 . ascaris clavata. La Cellule 1887;3:276. 19. Ostergren G, Heneen K. A squash technique for chromosome morphological studies. Hereditas 1962;48:332-41.

20. Alexander MP. A versatile stain for pollen fungi, yeast and bacteria. Stain Technol 1980;55:13-8. 